\title{
The Application of Zen Culture in Space Design CHEN Ming ${ }^{1, a}$, RAN kailong ${ }^{1, b}$
}

${ }^{1}$ Room306 ,Department of architecture, Wuhan University of Technology, Hubei, Wuhan, China

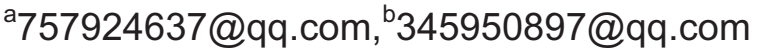

\section{Keywords: Zen culture; Space design; Create elements; Design Techniques}

Abstract. Architectural space design not only pays attention to the practicality, but also increasingly focuses on the design and infiltration of the cultural concept of space. Adding the cultural background to the space design, on the one hand can enhance the connotation of space, and on the other hand, can achieve cultural heritage. This paper briefly studies the application of "Zen space" in modern architecture, through analyzing the scale, shape, texture, color and other design elements of Zen space in architecture, and summarizes the theories and methods used in creating the Zen space, thus explores the significance of Zen space formation

\section{Introduction}

In recent years, the historical value of traditional culture attracts people's attention again, which has brought a popularity of backing traditional space life. As a representative of eastern "language", "Zen" has been taken seriously by more and more people. Nowadays, the objective space in reality is bustle and the fast-pace life brings people much invisible pressure so that people are eager to back to nature. We apply the cultural thought of Zen to the space deign, which can not only express such requirements but conforms to the natural instinct of human aesthetic competence.

\section{Zen Culture and Zen Space}

\subsection{Zen Culture}

"Zen"is transliterated from a Sanskrit "Dhyana", which means "quiet thinking" and "thinking and acting"and is seen as a thinking model aimed at quietness or a meditation in a peaceful and calm emotion. The Zen stems from Old India and it is a way of Sadhana in Buddhism The Zen tells people to sit in meditation, practice meditation, scan themselves and to abandon distracting thoughts so that people can reach a carried spiritual state. Zen Culture is a very abstract concept. It is based on the Zen thoughts and traditional eastern aesthetics and needs the "nature discovery". It lets people feel the existence of this realm in order to deliver a peaceful, quiet and fantastic mind to people, which make Zen closer to the art.

\subsection{Zen Space}

The architectural concept "dependent origination" of Zen is not only confined to a single pattern of construction but shows the diversity of patterns; the environmental view of Zen is based on the carried spiritual state and emphasizes on the integration of architecture and nature. These make the Zen express abstractly by using of natural elements in the space design of architecture and create a connection between humans and materials so that it can make everything in nature become a tool for passing the Zen. What's more, the space is an architectural essence centralized human on behalf of the appearance of materials. Zen Space can be seen as an architectural space equipped with "thought" and "soul" and it focus on building Zen. It realizes an 
inspiration of coexistence of the external looks and inner thoughts by using of integration of inner and external elements. Moreover, this architectural space not only integrates regionalism and national characters but inherits the essence of Eastern traditional culture. It also uses the spiritual conception "enlightenment and liberation" of Zen to inspect the connection between human and space so that it achieves the balance of material and spirit.

\section{The features of Zen Space}

\section{1 the central thought "intangible"}

The Zen urges the world view "No establishment of words, meaning counts more than configuration, extreme simplicity". The Zen thinks that people can be enlightened as long as they stay away from all text illusion. This "extreme simplicity" view belittles the natural pattern of things themselves and focus on finding the essence of them, which is so-called "empty". The "intangible"of Zen plays an important role in creating a spatial conception. It is different from "empty". The intangible design faces the inner world and simplifies the appearance as much as possible. It reduces much more decoration and mainly lets people feel the spiritual movement.

\section{2 the form "simple and unadorned" of manifestation}

The Zen purses a free, unconventional and simplified state. Apart from creating an intangible conception, the Zen uses its natural feature "simple and unadorned" to influence environment. Because of this kind of spatial design, the Zen space needs to obey the rule called simplification, freshness and elegance to embody the objective essence sufficiently. Under the influence of this conception, the spatial design should use the principle- simple but not single or unadorned but not Luxurious. The nature has offered us numerous materials of creation while the Zen Culture offers us a direction to find these materials. We should keep the initial beauty and frugality of these materials, such as mountains, stones and woods, etc. Designers elaborately design stones, woods or mountains on basis of obeying cultural essence and core. They starts from the whole conception and combine the natural beauty and textural beauty of those materials and spatial design together so that they can realize a visual effect "integration"and a spiritual conception and create an infinite space and a significant meaning.

3.3 the returned essence "nature is beauty"

Nature is seen as an important carrier of aesthetic and has always been an objective pursued by people. The "nature" of Zen focuses on the human enlightenment after they have a connection with the nature. It urges that people should find their inner world instead of the essence of nature and people should coexist with nature harmoniously. Therefore, people and nature should reach a harmonious state. Zen Space is seen as a carrier of expressing the Zen so that it should integrate the space with the natural beauty by virtue of everything in nature in order to create an elegant and refreshing atmosphere and that is the so-called regression of nature.

\section{The Zen Penetration in the Space Design of Architecture}

The modern space design is more likely to pursue a promotion of spatial conception and natural harmony. To some extent, it embodies the promotion of modern people's educational level and becomes a new objective that people pursue in every aspect of life. Zen Culture, which can satisfy the spiritual needs of humans, is equipped with many wonderful feelings such as quietness ,activeness and harmony. 
However,during the process of space design, the way of space design seems to be a very essential part to fix these problems such as how to embody the whole beauty and how to create a peaceful and harmonious atmosphere by using of Zen conception.

4.1 the principle of space design

1) natural harmony

The Zen urges the harmonious state between human and nature. It pursues the integration of human and nature and the integration of objectives and humans. It can be called beauty when the connection between human and nature reaches a balanced state. The balance here refers to a dimension or a standard and it emphasis on the existence, penetration and harmony of kinds of elements and strengths and it urges the way of integration. In the space design,the balance is embodied in shape,color,light and quality. We make every architectural space reach a balanced state by handling and adjusting the connection between surface, quality of materials,color and light.

2) simplifying

The Zen space has its own special charm and the design of every detail is planned by designers elaborately. The "minimalism" has been running through eastern culture for thousands of years. "less is more" is taken as a main thought which requires designers have their unique understanding of Zen Space. The "less"presentations ,the "more" connotations and more imaginary space can be offered. This special space is not just simple so that it can offer people a better meditation. In this simple state, the participants can meditate the spatial spiritual of Zen in that the simple state embodies the essence of material.

\section{2 elements of design}

Generally speaking, the connotations expressed by the space are mainly based on the subject setting and the expression of subject is the result of combined actions of many elements, for example the dimension and proportion of space, the color environment, the quality of materials and the lights, etc.

Different spaces usually bring people different feelings. We should take the emotional infection into consideration and expand the dimension of space on the premise of ensuring the using function. Especially in terms of buildings with religion and cultural essence, they pay more attention to the integration of requirements and spirits. The enclosed space brings people pretty, peaceful and imitate atmosphere. On the contrary, the open space can interact more with the outside world and integrate with nature.

The quality of materials leads the mental feeling of human subjectively. For example, people can feel the strict and cold character of sand and stone and feel the warm and gentle character of wood materials and feel the strength of metal material. The quality of these materials can create a more conceptual space feeling by different matches.

During the process of creating the spatial conception, the color is an essential element. In the space experience, visual sense has taken a main part in that most information is gained by the visual feedback. The color itself is not influenced by the outside world but it can bring people different emotional experiences. We can usually bring people different feelings on visualization and create more distinctive spatial conceptions by using colorific temperature,sense of movement and sense of massiveness.

The artistic effect of architectural space needs to be heightened by the light. The use of natural light and artificial light has a huge effect on the creation of artistic conception. The existence of shadow brings the space a more changeable conceptual 
effect. Different shapes of light make the space more conceptual. It can bring people different feelings by using different ways, such as refraction, reflection, projection and scattering.

The influential factors of design of Zen Space are far more than above. As long as we take good use of these basic elements to process the space in division and artistic rendering, we can build the spatial conception in numerous ways.

\section{The Practical use of Zen Space}

\subsection{Zhen Junei Long Ye Cemetery (1) Big Head Buddha}

The architectural work from Tadao Ando has a deep meaning of Zen,which is close related to his design conception : first, the "people oriented" conception; second, the undivided character of human and nature. His works bring people an inner peace of architecture again.

Zhen Junei Long Ye Cemetery inherits the most common feature of Ando's building.The Buddha mostly hided in visuals has its own holy space. You can only see the head of this Buddha in any directions under the wide mountain of lavender in that Ando uses space and shadow and natural elements skillfully to create the space with extreme sense of ceremonial. The cemetery's owner originally centralized the Buddha and rebuilt this place as a landmark. However, Ando told him that if it is buried, it can represent its intrinsic scale. Therefore, this "growing with nature"-themed architecture was built.
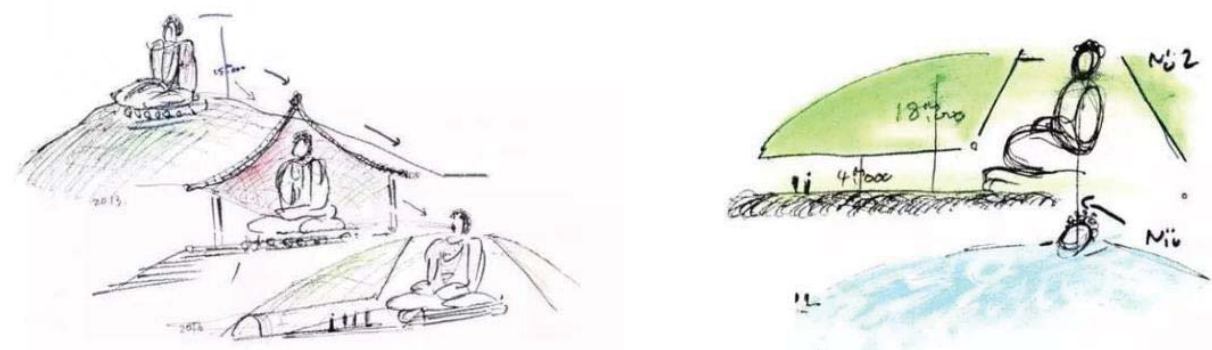

Figure 1,2 Ando design ideas manuscript

Water is the most favorable element for Ando. On the way to visit the big Buddha, visitors have to pass a water garden measured $16.2 \times 61.2 \mathrm{~m}$ which cuts down the way to go to the Buddha. Therefore, visitors have to detour it from the two sides. This design not only increases a sense of mystery but brings a sense of ceremony by virtue of the flowing water. Visitors have to pass a $40 \mathrm{~m}$ length passageway through the water pool. The material of the passageway is a keen material for Ando ---bare concrete. Later, the Buddha begins to appear as you pass the passageway. Visitors view through the Buddha from its feet to head and sit quietly in this uncovered space, which makes the whole space sacred.

When you detour the water poor and pass through the gloomy passageway, the faith comes with light. Ando creates such sacred space by using of circulated space and natural elements .A strong sense of Zen appears, which can be seen as the initial pursue of Zen. 

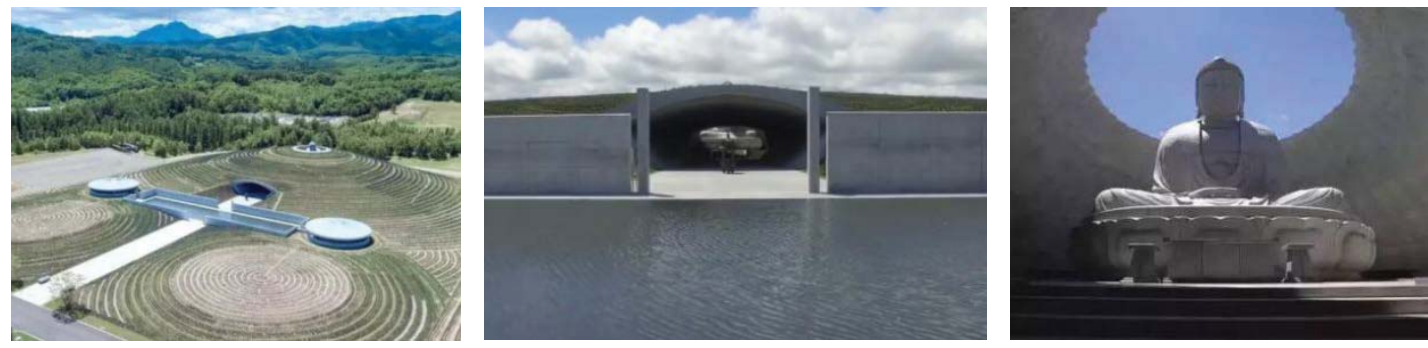

Fig. 3 aerial view

Fig.4 through the water, corridor can be seen Buddha Fig.5 sitting Buddha solemn

\subsection{The Church Of Light}

The Church Of Light is known as one of the most representative Zen buildings. The main part of this church is a rectangle made of bare concrete and is divided into two parts in $15^{\circ}$ by a single piece of wall. The bigger part is the church and another is entrance. This simple and slant wall not only enriches the space orders but makes the whole space multiplied. The notable "cross of light" is on the back wall of the platform of this church.

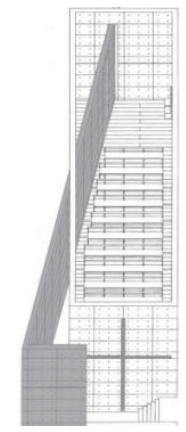

Fig.6 The sloping wall divides the space

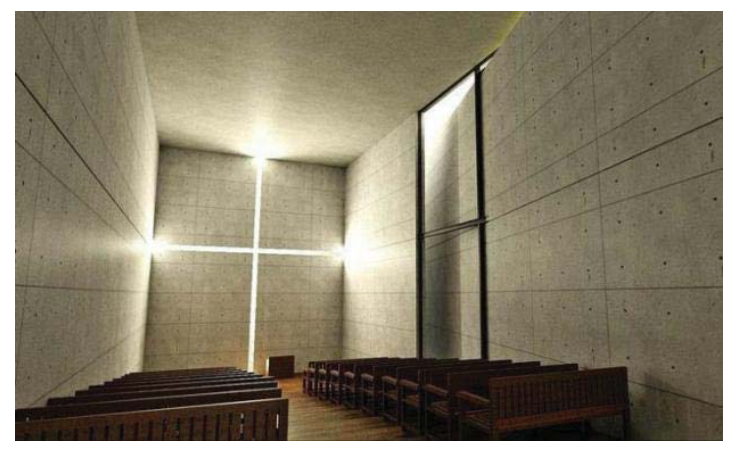

Fig.7 cross-ray makes the space full of sense of ritual

The light comes into the space under the scrupulous direction of Ando in accordance with the gentle light reflected by the wall made of $15^{\circ}$ concrete, which not only fixes the problem that the wall cannot be adapt to window but dissociates the architectural space from the bustle outside world. Moreover, the architectural wall is made of plain concrete and it keeps the original quality of materials, letting people feel the reality of this architecture. The whole space is pure and simple and it brings the rethink of Zen.

\subsection{Fagu Mountain Agricultural Zen Temple Shui Yue Dojo (2)}

A master of modern architecture named Yao Renxi whose architectural works have significant thoughts of Zen. His works in recent years are all involved in the Zen. Shui Yue Dojo is the most representative one. As you are walking into the Dojo, you can see the main lecture room standing inside the $80 \mathrm{~m}$-long lotus pool. The lotus pool has a clear surface. The enormous pillar' $\mathrm{s}$ inverted image is reflected by blowing curtain which brings the whole building a sense of mystery. These experiences of special spaces are more likely to be a present of nature. The material of the main part of this building is concrete made of water in order to show the natural quality of the material and express the simple and unadorned meaning of Zen.

The design of the lower half of this hall deliberately uses the transparent material in order to bring the upper half - wooden "box" a magic impression. The wooden 
wall of the west architecture is engraved with The Heart Sutra. When the sunlight penetrates in the building, the space seems to be filled with intelligence as if showing people the meaning of Zen. Although the building seems to be a simple architecture, the content of its inner space is so colorful and various. Every highlight of this building is excellent-designed by the designer, which is praised by people sincerely.

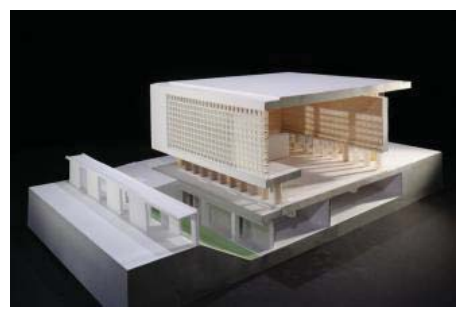

Fig.8 the Model

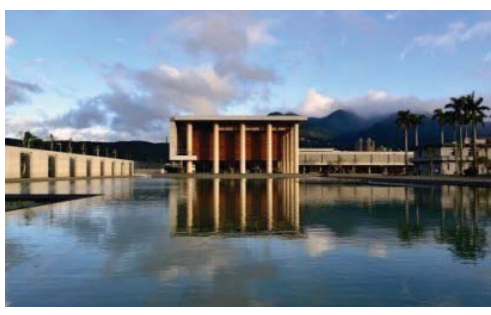

Fig.9 Architectural reflection in water

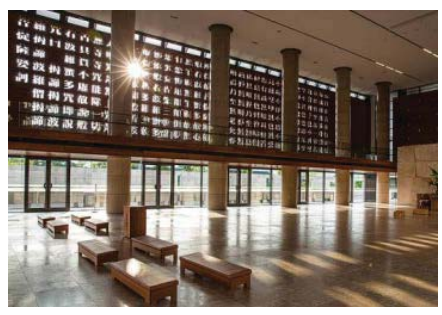

Fig.10 Sun through the hollow text

Of course, the varieties of Yao Renxi' s representative works are not only limited to Shui Yue Dojo. They also include Wu Town Theater,Wu Tai Mountain Zen Center and etc. The essences of these buildings are all hidden under the simple outlooks. The buildings show up their meaning of Zen in every detail in front of people by using of complicate spatial relations, natural feature of materials and ingenious skills.

\section{4 the commune at the foot of The Great Wall---Bamboo House}

The Bamboo House uses a large amount of natural material -- bamboo so that it makes a good integration of architecture and environment, which is closely related to the architectural conception "let it fade away" of the designer named Wei Yanwu. One of the important ways for building a space of Zen is using the natural features of materials and making a close connection between the space and the nature. The dimension of the whole building is delicate and well embodies the using of natural materials.

The wall outside the Bamboo House consists of a huge glass wall and slim bamboos, which lets the sunlight penetrate inside the wall in any angles. The light is dissociated by bamboos so that the room seems to be filled with sunshine. All sides of the tea room are made of bamboos. The tea room floats on water surface and you can see the Great Wall vaguely through gaps of bamboos. The strong atmosphere of Zen spreads all sides of the room. The inside furniture is modern but caters to the room perfectly. Seat chairs are made of bamboos and there are potting and cattail hassock made in Japanese style inside the room. Every detail of this house spreads the meaning of Zen.

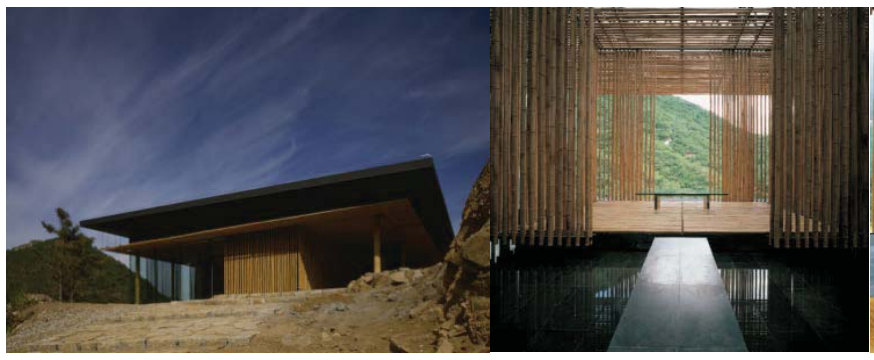

Fig.11 bamboo house appearance
Fig.12 indoor tea room

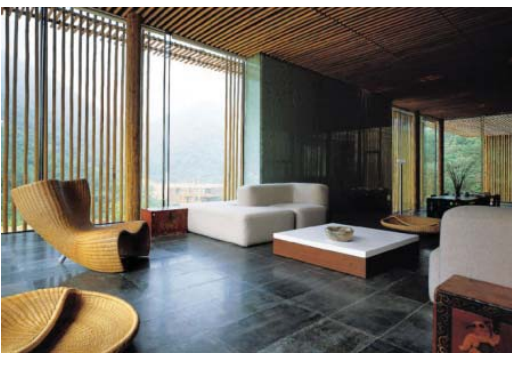

Fig.13 full of Zen furniture decoration 


\section{Summarize}

All in all, the process of building a Zen space is mainly based on nature and integrates the beauty of spatial form and nature, which can realize the integration of human and nature. The architecture tends to express the meaning of Zen with the most natural and simple outlooks. It should not have various decorations. As designing a Zen space, the designers must be equipped with a heart of Zen and analyze the consisting elements of the space and then select the subject of the space. The space should integrate with traditional aesthetics and create a spatial artistic conception with a strong spirit of Zen so that it can offer users a place of insight, which is exactly the design objective of modern Zen Space.

\section{References}

[1] Daisetz Suzuki. Zen and life . Liu dabei, Meng Xiangsen, trans.Mount Huangshan publishing house, Hefei,2010,171-179.

[2]Yun yajie.Application of Oriental Zen Culture in Space Design.J.Art Literature, 2014(01) 180-181.

[3] Qin Yaping.The beauty of the space of Zen - the application of Zen aesthetics in modern space design .J. Interior Design, 2011(03)17-19+33+20.

[4] Wang Yujia. Zen culture in the interior design of the research and application.J. Modern decoration (theory), 2014(09)48-49.

[5] Meng Xiaohui, Jin Ya. Analysis of the beauty of Zen in the application of modern interior space design.J. beauty and times , 2013(09)82-83.

[6] "Master Series" editorial department. Ando Tadao's works and thoughts.China Electric Power Press,Beijing, 2005.

Figures 1, 2, 3, 4, 5 are derived from http://www.aiweibang.com/

Figures 6,7 are derived from the "Tadao Ando works and thoughts"

Figure 8 is derived from http://award.kinpan.com/

Figures 9,10 are derived from

http://www.krisyaoartech.com/gb/projects/spiritual-spaces/WATER-MOON-MONAS TERY

Figures 11,12,13 are derived from http://bulo.hujiang.com/ 